$1-1-1986$

\title{
Plasmons in semiconducting superlattices with a complex unit cell
}

Robert A. Mayanovic

Gabriele F. Giuliani

John J. Quinn

Follow this and additional works at: https://bearworks.missouristate.edu/articles-cnas

\section{Recommended Citation}

Mayanovic, Robert A., Gabriele F. Giuliani, and John J. Quinn. "Plasmons in semiconducting superlattices with a complex unit cell." Physical Review B 33, no. 12 (1986): 8390.

This article or document was made available through BearWorks, the institutional repository of Missouri State University. The work contained in it may be protected by copyright and require permission of the copyright holder for reuse or redistribution.

For more information, please contact BearWorks@library.missouristate.edu. 


\title{
Plasmons in semiconducting superlattices with a complex unit cell
}

\author{
Robert A. Mayanovic and Gabriele F. Giuliani \\ Department of Physics, Purdue University, West Lafayette, Indiana 47907 \\ John J. Quinn \\ Department of Physics, Brown University, Providence, Rhode Island 02912
}

(Received 25 November 1985)

\begin{abstract}
We discuss the theory of the intrasubband longitudinal electronic excitations in type-I semiconducting superlattices in which the repeat unit is comprised of two electronic layers. The spectrum of a semi-infinite superlattice of this new type consists of both bulk- and surface-plasmon branches. For each value of $k$, the wave vector along the layering direction, there are two bulk plasmons. As $k$ is varied at a given in-plane component of the wave vector the frequencies of these excitations form two separated continua. The surface plasmons correspond to collective excitations which are localized near the interface with an insulator. Their dispersion relation lies outside of the bulkplasmon continua. These modes have the peculiarity of being intrinsically free of Landau damping. We find that in these systems acoustic surface plasmons always exist if the distance between the first two layers is more than half the superlattice spacing.
\end{abstract}

\section{INTRODUCTION AND DISCUSSION}

The electronic dynamics in semiconducting superlattices $^{1}$ (SL's) has recently attracted much theoretical interest. SL's are artificially layered structures which, in a first approximation, can be viewed as a series of equivalent quantum wells in which the motion of the carriers is subject to space quantization. Semiconducting SL's are generally referred to as being of type I or type II, depending on the type of carriers trapped in their quantum wells. In type-I SL's, the carriers in the quantum wells are electrons. This type of SL is typified by the familiar $\mathrm{GaAs} / \mathrm{Al}_{x} \mathrm{Ga}_{1-x}$ As heterostructure. In type-II SL's, electronic layers alternate with hole layers. The heterostructure $\mathrm{GaSb} / \mathrm{InAs}$ provides an example of a type-II SL. In a SL, the single-particle spectrum for motion along the layering direction is characterized by a series of subbands corresponding to the discrete quantum-well states. The motion in the plane of the layers is instead practically two dimensional.

Electronic collective excitations in SL's are referred to as intersubband or intrasubband excitations, depending on whether or not transitions between subbands are involved. The bulk collective excitations of an infinite SL of type I and type II have been studied both theoretically ${ }^{2-6}$ and experimentally by a number of authors. ${ }^{7-9}$

More recently, a new type of surface plasmons, which are localized near the interface of a semi-infinite superlattice with an insulator, have been introduced. ${ }^{10}$ These excitations, at variance with the familiar case of surface plasmons in ordinary metals, ${ }^{1-13}$ have the peculiarity of being free of Landau damping and could prove of interest in the field of surface-wave devices. A large variety of surface-plasmon branches are found in both type-I and type-II SL's. ${ }^{10,14-17}$ The effects of intersubband transitions $^{5,6}$ and the Raman scattering amplitude ${ }^{18}$ of these plasma modes have recently been investigated for the case of type-I SL's. A more complete discussion of the general theory of surface plasmons in SL's can be found in Ref. 19. In type-I SL's, surface plasmons only exist for finite values of the in-plane wave vector $q$ and decay into bulkplasma modes in the long wavelength limit. ${ }^{10}$ This feature makes it difficult to couple to them via light scattering techniques. In type-II SL's, on the other hand, more than one branch of surface plasmons can exist at the same value of $q$ and, in suitable circumstances, the system will admit acoustic surface plasmons. The conditions for the existence of acoustic surface plasmons in type-II SL's are discussed in Refs. 15 and 17. In this case, a crucial role is played by the values of the electron and hole concentration in the layers.

In this paper, we investigate the excitation spectrum of a type-I SL in which the repeat unit is comprised of two electronic layers instead of just one. ${ }^{20}$ The repeat scheme is of the type $a b-a b-\cdots$, where $a$ is the distance between the two layers in the unit cell, and $c=a+b$ is the superlattice spacing. A schematic diagram of this system is shown in Fig. 1. This new kind of type-I semiconducting SL is as easy to fabricate as the usual type. The reason to introduce such a slight complication lies in the fact that, as will be shown below, this SL has a rich excitation spectrum that also admits acoustic surface plasmons. At variance with the type-II SL's, however, the relevant parameters determining the spectrum are simply of geometrical character. In particular, we find that when the distance between the first and second layer is larger than half the superlattice spacing (i.e., if $a>b$, as in Fig. 1, for instance), acoustic surface plasmons always exist. We suggest that this type of SL is most appropriate for the observation and characterization of surface plasmons in semiconducting SL's. It is also interesting to notice that the group velocity of these modes (see below) is also strongly dependent on geometrical factors. It is then quite obvious how, in view of the current flexibility in design and 


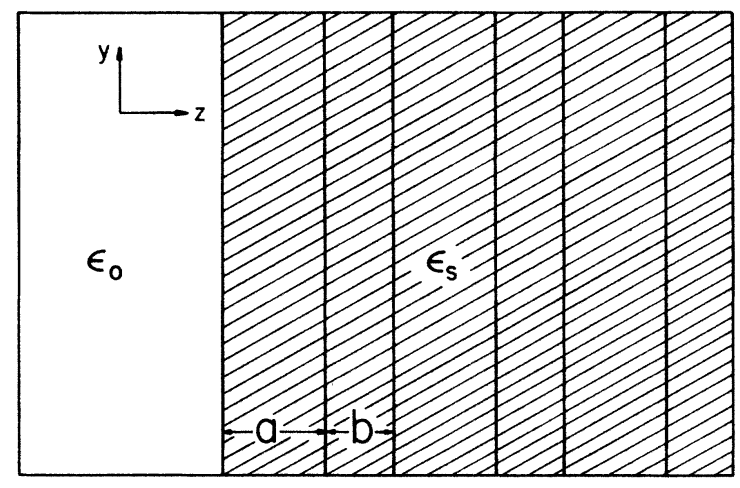

FIG. 1. Schematic diagram of the $a b-a b_{-} \cdots$ type-I superlattice discussed in the text. The electronic layers are embedded in a semiconducting medium of dielectric constant $\epsilon_{s}$. In each layer, the electrons move in the $x, y$ plane. The heterostructure is terminated at the interface $(z=0)$ with an insulating medium of dielectric constant $\epsilon_{0}$. In general, $\epsilon_{s}$ and $\epsilon_{0}$ will depend on $\omega$.

manufacture of semiconducting SL's, it could be very simple to devise situations in which the surface waves have the required properties.

\section{BULK PLASMONS}

Our model of an infinite SL consists in a periodic array of electronic layers stacked along the $z$ direction, as exemplified in Fig. 1. For simplicity, we will assume here that in each layer one has a strictly two-dimensional electron gas. $^{21}$ The layers are embedded in a host insulating medium characterized by a dielectric constant $\epsilon_{s}$. In general, $\epsilon_{s}$ will depend on the excitation frequency $\omega$. Our approach is patterned after the discussion of Refs. 10 and 19. From Maxwell equations, one can readily express the electric field $\mathbf{E}$, associated with an electronic excitation frequency $\omega$, as follows: for $m c \leq z \leq m c+a(c=a+b$, and $m$ is an integer),

$$
\begin{aligned}
\mathbf{E}=e^{i(q y-\omega t)} & \left(0, E_{m}^{+} e^{i \beta(z-m c)}+E_{m}^{-} e^{-i \beta(z-m c)},\right. \\
& \left.-\left(E_{m}^{+} e^{i \beta(z-m c)}-E_{m}^{-} e^{-i \beta(z-m c)}\right) q / \beta\right),
\end{aligned}
$$

and for $m c+a \leq Z \leq m c+c$,

$$
\begin{aligned}
\mathbf{E}=e^{i(\boldsymbol{q y}-\omega t)} & \left(0, F_{m}^{+} e^{i \beta(z-m c-a)}+F_{m}^{-} e^{-i \beta(z-m c-a)},\right. \\
& \left.-\left(F_{m}^{+} e^{i \beta(z-m c-a)}-F_{m}^{-} e^{-i \beta(z-m c-a)}\right) q / \beta\right),
\end{aligned}
$$

where $\beta=\left(\epsilon_{s} \omega^{2} / c^{2}-q^{2}\right)^{1 / 2}, q$ being the component of the wave vector of the wave in the $x, y$ plane. $E_{m}^{\ddagger}$ and $F_{m}^{ \pm}$are suitable field amplitudes. The bulk-plasmon dispersion relation can most easily be obtained by imposing the periodicity conditions

$$
E_{m}^{ \pm}=e^{-\alpha m c} E_{0}^{ \pm}
$$

and

$$
F_{m}^{ \pm}=e^{-\alpha m c} F_{0}^{ \pm}
$$

where $\alpha$, a complex wave vector to be determined, describes the variation of the field along the layering direction. The coefficients $F_{m}^{+}$and $F_{m}^{-}$can be expressed in terms of $E_{m}^{ \pm}$by requiring that $E_{y}$ be continuous at $z=m c+a$, and that the discontinuity of $D_{z}$ at $z=m c+a$ be equal to $4 \pi \rho, \rho$ being the induced electronic layer charge density at $z=m c+a$. Next, the ratio $E_{m}^{+} / E_{m}^{-}$can be obtained by imposing that $E_{y}$ be continuous at $z=m c$. This quantity can also be evaluated by demanding that the discontinuity of $D_{z}$ at $z=m c$ have the appropriate value. The two different expressions are then equated. After some algebraic manipulations, this consistency relation can be expressed as follows:

$$
\begin{aligned}
D_{B}(\omega)= & \left(\gamma^{2} / 4\right)\{\cosh (i \beta c)-\cosh [i \beta(b-a)]\} \\
& -\gamma \sinh (i \beta c)+\cosh (i \beta c)-\cosh (\alpha c)=0,
\end{aligned}
$$

where

$$
\gamma(q, \omega)=4 \pi i e^{2} \beta \chi_{0}(q, \omega) / \epsilon_{s} q^{2}
$$

and $\chi_{0}(q, \omega)$, within the random-phase approximation, is the susceptibility of a noninteracting two-dimensional electron gas. ${ }^{22}$ Equation (3) is the dispersion relation for the bulk plasmons. A direct inspection of Eq. (2) leads to the conclusion that in an infinite SL, if a finite value for the electric field is demanded, the wave vector $\alpha$ is purely imaginary. In this case, we can write $\alpha=i k$.

A simple analytic expression for $\omega(q, k)$ can be obtained in the nonrelativistic limit $(i \beta \rightarrow-q)$ when $\omega \gg>q v_{F}\left(v_{F}\right.$ is the Fermi velocity of the electrons in the quantum well $)^{23}$ and $\epsilon_{s}$ and $\epsilon_{0}$ are taken to be constants. In this case, one can make use of the approximation $\chi_{0}(q, \omega) \approx n q^{2} / m^{*} \omega^{2}$, with $n$ being the number of electrons per unit area and $m^{*}$ the electronic effective mass in the layers. ${ }^{24}$ The solution of Eq. (3) leads, in this case, to the following result:

$$
\begin{aligned}
\omega_{ \pm}^{2}(q, k)= & \frac{1}{4}\left\{\left[\omega_{p}^{2} q c \sinh (q c) /[\cosh (q c)-\cos (k c)]\right\}\right. \\
& \times\left[1 \pm\left(1-[\cosh (q c)-\cos (k c)]\{\cosh (q c)-\cosh [q(b-a)]\} / \sinh ^{2}(q c)\right)^{1 / 2}\right],
\end{aligned}
$$

with $\omega_{p}^{2}=8 \pi n e^{2} / m^{*} c \epsilon_{s}$. A plot of $\omega_{ \pm}$versus $q$ for a particular value of $k$ is shown in Fig. 2. Notice that for each value of $q$, there are two possible acoustic bulk plasmons. As $q c$ increases, both branches merge with $\frac{1}{2} \omega_{p}(q c)^{1 / 2}$, the excitation frequency of an isolated layer. As $k$ is varied, the bulk excitation frequencies form two separated continua, as shown in Fig. 3. All the bulk plasmons have an acoustic behavior for $q c \rightarrow 0$, with the only exception being the $k=0$ plasmon. This should be compared with the corresponding continua in type-I and type-II SL's. ${ }^{19}$ 


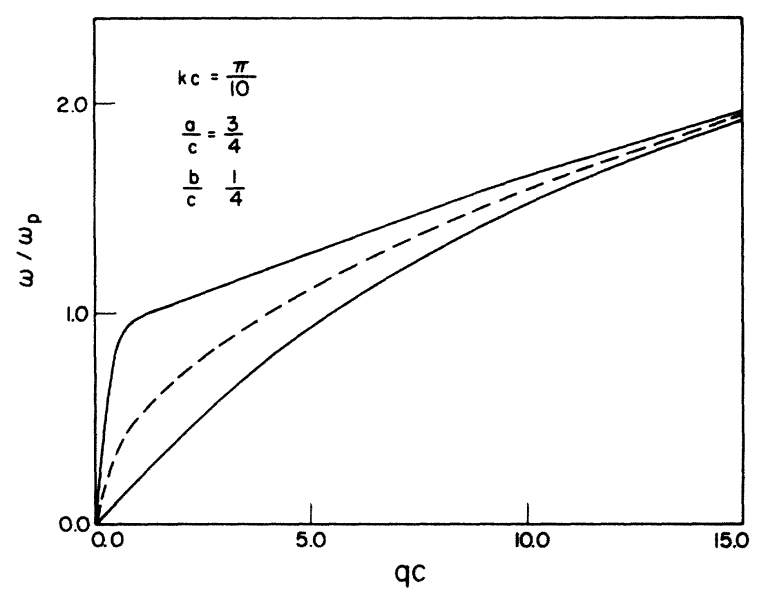

FIG. 2. Plot of frequency $\omega$ versus $q$, the in-plane wave vector, for the plasma excitations of an infinite semiconducting superlattice of the type discussed in the text. The value of $k c$ is $\pi / 10$. The dashed line corresponds to the plasma frequency of an isolated layer. The electron-hole continuum, if included in the plot, would lie considerably lower than the two plasmon branches.

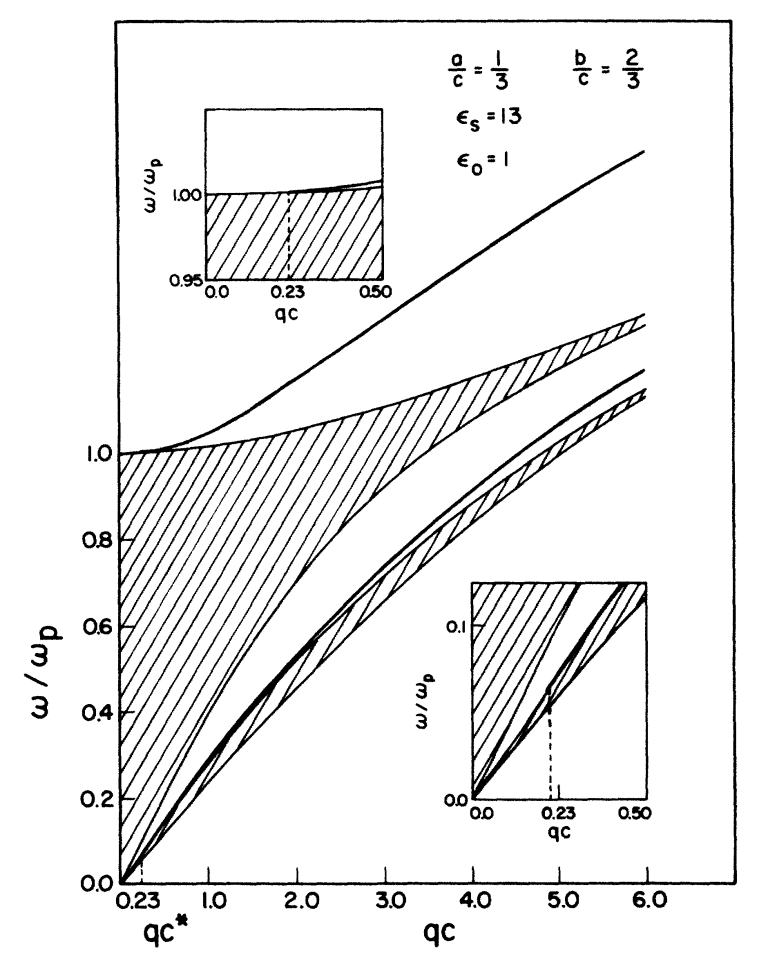

FIG. 3. Dispersion relation $\omega$ versus $q$ for the plasma excitations of a semi-infinite $a b-a b-\cdots$ type-I semiconducting SL discussed in the text. The two shaded regions represent the bulk-plasmon-band continuum. The two thick lines correspond to the surface-plasmon branches. The top and lower insets show expanded plots of the small-qc region of the top and lower branches, respectively. The values of the parameters are as follows: $a / c=\frac{1}{3}, b / c=\frac{2}{3}, \epsilon_{s}=13, \epsilon_{0}=1$. Notice that both surface excitations merge into the bulk-modes continuum at $q^{*} c=0.23$. No surface plasmons are present in the longwavelength limit.
The frequencies in the upper continuum are given by $\omega_{+}(q, k)$, with $k$ ranging between 0 (the upper edge) and $\pi / c$ (the lower edge). The frequencies in the lower continuum can instead be conveniently expressed as $\omega_{-}(q, k)$, where $k$ ranges between $\pi / c$ (the upper edge) and $2 \pi / c$ (the lower edge). This description, (although redundant) allows one to recover easily the type-I SL situation when $a \rightarrow b$. In this case, the value of $k$ corresponding to the lower edge of the continuum [there is only one bulkplasmon continuum in a type-I SL, as $\omega_{-}(q, \pi / c)$ $\left.\rightarrow \omega_{+}(q, \pi / c)\right]$, is $2 \pi / c \rightarrow 2 \pi / 2 a \rightarrow \pi / a$, as it should be. ${ }^{16,19}$ It is interesting to comment here on a different limiting behavior: If $a$ is kept constant as $b$ goes to infinity, the system reduces to an isolated pair of electronic layers. In this case, Eq. (5) gives

$$
\lim _{q b \rightarrow \infty} \omega_{ \pm}^{2}(q, k)=\left(2 \pi n e^{2} / a m^{*}\right) q a\left(1 \pm e^{-q a}\right),
$$

with the following limiting behaviors:

$$
\begin{aligned}
& \lim _{q a \rightarrow 0}\left(\lim _{q b \rightarrow \infty} \omega_{+}^{2}\right)=\left(4 \pi n e^{2} / m^{*}\right) q, \\
& \lim _{q a \rightarrow 0}\left(\lim _{q b \rightarrow \infty} \omega_{-}^{2}\right)=\left(2 \pi n e^{2} / a m^{*}\right)(q a)^{2},
\end{aligned}
$$

in agreement with the results of Ref. 25.

\section{SURFACE PLASMONS}

Consider a semi-infinite structure of the type represented in Fig. 1. At $z=0$, the SL is terminated at the interface with a different insulating medium characterized by a dielectric constant $\epsilon_{0}{ }^{26}$ Similarly to $\epsilon_{s}, \epsilon_{0}$ will, in general, depend on the excitation frequency $\omega$. Equations (1a), (1b), (2a), and (2b) for the electric field are still valid for $z>0$ (i.e., $m=0,1,2, \ldots$ ). For $z<0$ we have, instead,

$$
\mathbf{E}=E_{0} e^{i(\boldsymbol{y}-\omega t)} e^{\beta_{0}{ }^{2}}\left(0,1,-i q / \beta_{0}\right), \quad z \leq 0
$$

with $\beta_{0}=\left(q^{2}-\epsilon_{0} \omega^{2} / c^{2}\right)^{1 / 2}$. It is important to notice here that we can still make use of Eqs. (2a) and (2b) for positive values of $m$. In this case, however, we expect to find that in order to describe surface excitations, $\alpha$ must be a complex wave vector with a positive real part. The boundary conditions discussed in the preceding section are still valid for positive integers $m$ but must now be supplemented with the conditions allowing for the continuity of $E_{y}$ and for the appropriate value of the discontinuity of $D_{z}$ at $z=0$. It is interesting to remark that by following the procedure outlined in the preceding section, it is possible to derive several different (but totally equivalent) dispersion relations for the surface plasmons. Of all the possible formulas, however, we have found the following relationships particularly simple to use:

$$
\begin{aligned}
D_{S}(\omega)= & {\left[\cosh (q b)-\sinh (q b) / \epsilon_{R}\right] } \\
\times & {\left[A_{+} e^{q c}+A_{-} e^{-q c}\right.} \\
& \left.+\gamma \sinh (q b)\left(A_{+} e^{q a}+A_{-} e^{-q a}\right)\right] \\
& -A_{+} e^{q a}-A_{-} e^{-q a}=0,
\end{aligned}
$$

with $\alpha$ given by 


$$
e^{-\alpha c}=\frac{\frac{1}{2}\left(A_{+} e^{q a}+A_{-} e^{-q a}\right)}{\cosh (q b)-\sinh (q b) / \epsilon_{R}} .
$$

In Eqs. (8) and (9),

$$
A_{ \pm}(q, \omega)=1 \pm\left[i \beta / \beta_{0} \epsilon_{R}-\gamma(q, \omega)\right],
$$

and we have defined the ratio $\epsilon_{R}=\epsilon_{s} / \epsilon_{0}$.

Equations (8) and (9) can be readily solved by numerical methods to give the desired surface-plasmon dispersion relation. Care must be taken, however, to discard spurious solutions corresponding through Eq. (9) to negative values of $\operatorname{Re}(\alpha c)$. As noted above, the boundary conditions discussed in the preceding section are still valid $(m>0)$, so that any solution of Eq. (9) must also satisfy Eq. (3). In this case, however, $\alpha$ will have both a suitably real and imaginary part.

As is the case for type-I and type-II SL's, ${ }^{14}$ the dispersion relation can be obtained in an analytic form in the nonrelativistic limit $(i \beta \rightarrow-q)$ when $\omega \gg q v_{F}$ and $\epsilon_{s}$ and $\epsilon_{0}$ are taken to be constants. The result is

$$
\omega_{\mathrm{SP}}^{2}(q)=\omega_{p}^{2}\left\{\left[C_{1} \pm\left(C_{1}^{2}-4 C_{0} C_{2}\right)^{1 / 2}\right] / 2 C_{0}\right\},
$$

where the $C_{i}$ 's are defined as follows:

$$
\begin{aligned}
& C_{0}=(q c / 2)^{2} \sinh (q a)\left[\cosh (q b)-\sinh (q b) / \epsilon_{R}\right], \\
& C_{1}=\frac{1}{2} q c\left\{\left(1-1 / \epsilon_{R}^{2}\right) \sinh (q b) \sinh (q a)\right. \\
& \left.+2 \cosh (q a)\left[\cosh (q b)-\sinh (q b) / \epsilon_{R}\right]\right\}, \\
& C_{2}=\left(1-1 / \epsilon_{R}^{2}\right) \sinh (q c) \text {. }
\end{aligned}
$$

It is simple to verify that, for $b \rightarrow a$, the dispersion relation (11) reduces to the well-known analytic formula ${ }^{27}$ valid in the case of a type-I SL, i.e.,

$$
\begin{aligned}
\omega_{\mathrm{SP}}^{2}(q)= & \left(4 \pi n e^{2} / m^{*} \epsilon_{s}\right) q\left[\cosh (q a)-\sinh (q a) / \epsilon_{R}\right] \\
& \times\left[\left(1-1 / \epsilon_{R}^{2}\right) \sinh (q a)\right]^{-1}, \quad a=b .
\end{aligned}
$$

Various plots of the surface-plasmon dispersion relation are presented in Figs. 3-6. There are several general features in our results that are worth mentioning:

(a) Surface-plasmon modes exist only when their frequency lies outside the bulk-modes continua.

(b) The system admits two branches of surface-plasma modes: One branch, which we will refer to as the ordinary surface plasmon (OSP), always lies above (for $\epsilon_{s}>\epsilon_{0}$ ) or below (for $\epsilon_{s}<\epsilon_{0}$ ) the two bulk-modes continua. The second branch, which we will refer to as the extraordinary surface plasmon (ESP), always lies in the region of the $\omega, q$ space between the lower edge of the upper bulk-plasmon continuum and the upper edge of the lower bulk-plasmon continuum. It is the OSP that corresponds to the surface plasmon of type-I SL's and whose frequency for large $q c$ tends to $\omega_{P}\left[\frac{1}{2} q c /\left(\epsilon_{s}+\epsilon_{0}\right)\right]^{1 / 2}$, the frequency of an isolated electronic layer which is sandwiched between two regions of dielectric constants $\epsilon_{s}$ and $\epsilon_{0}$.

(c) As is well known, ${ }^{10}$ due to the space quantization along the layering direction, these surface excitations are practically free of the intrinsic Landau damping which is characteristic of the surface plasmons in ordinary three-

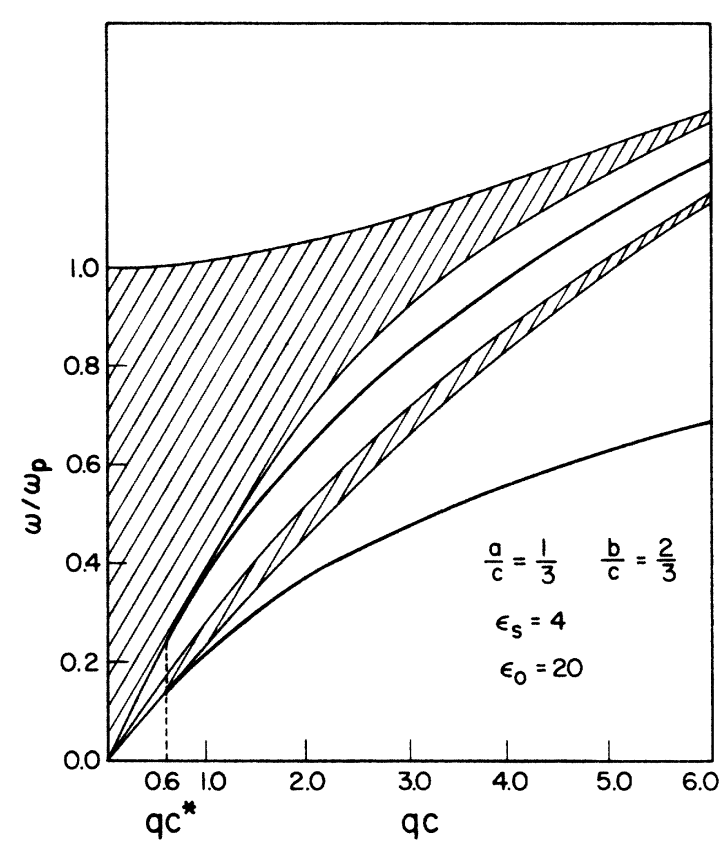

FIG. 4. Same as Fig. 3. The values of the parameters in this case are $a / c=\frac{1}{3}, b / c=\frac{2}{3}, \epsilon_{s}=4, \epsilon_{0}=20$.

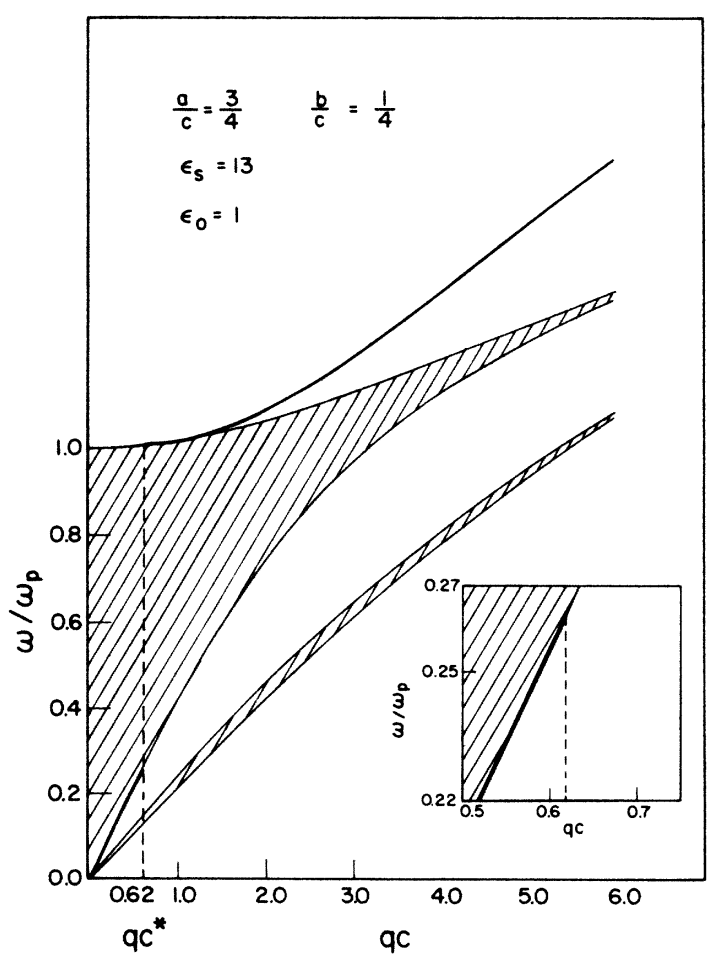

FIG. 5. Same as Fig. 3. The values of the parameters in this case are $a / c=\frac{3}{4}, b / c=\frac{1}{4}, \epsilon_{s}=13, \epsilon_{0}=1$. Here $a>b$, and in the long-wavelength limit, as shown in the inset, we have an acoustic surface plasmon with a group velocity given by Eq. (14) in the text. 


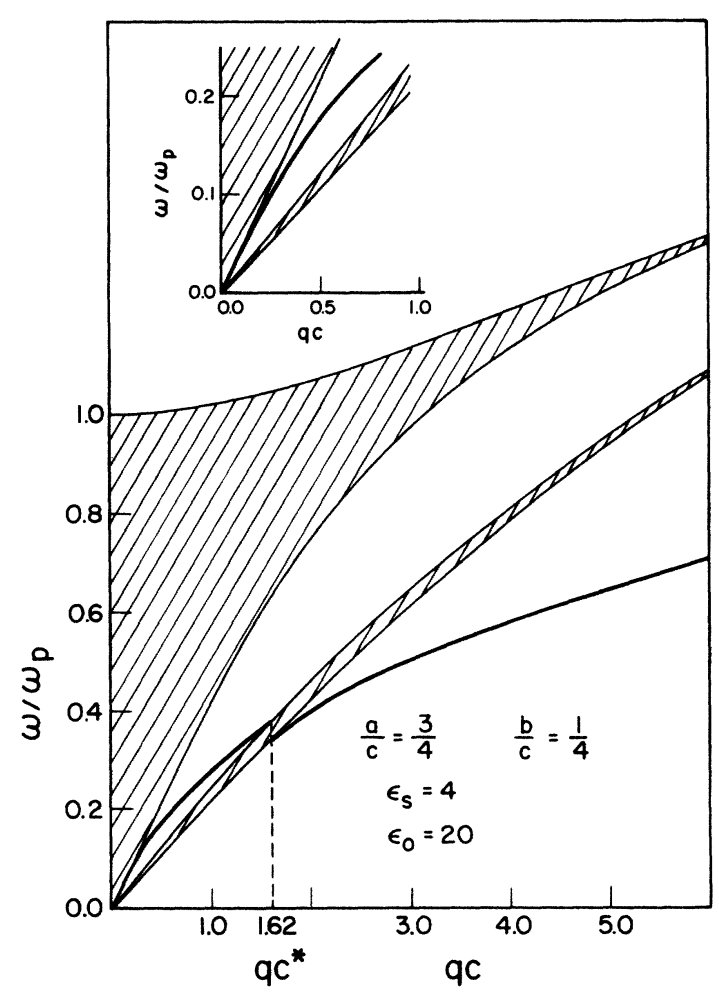

FIG. 6. Same as Figs. 3 and 5. The values of the parameters in this case are $a / c=\frac{3}{4}, b / c=\frac{1}{4}, \epsilon_{s}=4, \epsilon_{0}=20$.

dimensional metals. ${ }^{11-13}$

Depending on the geometry of the system, there are two distinct situations:

(i) For $b>a$ (Figs. 3 and 4), both the OSP and ESP branches always exist at the same value of $q$ for $q$ larger than a critical wave vector $q^{*}$ (see below) and merge at $q^{*}$ into the continua. In this case, there are no surfaceplasma modes in the long-wavelength limit.

(ii) For $a>b$ (Figs. 5 and 6), only one branch exists at a time for a given $q$. The OSP exists for large $q$ and merges into the continuum at $q^{*}$. The ESP emerges from the bulk-mode continuum at exactly $q^{*}$ and has an acoustic dispersion at $q \rightarrow 0$. In this limit, the ESP frequency is given by

$$
\begin{aligned}
\omega_{\mathrm{SP}}(q)= & \left(2 \pi n e^{2} / m^{*} \epsilon_{s}\right)^{1 / 2} \\
& \times\left\{a-b^{2} /\left[c\left(\epsilon_{R}^{2}-1\right)\right]\right\}^{1 / 2} q+O\left(q^{2}\right) .
\end{aligned}
$$

The coefficient of the linear term in Eq. (14) is $v_{S P}$, the group velocity of the acoustic surface wave, a quantity strongly dependent upon geometrical parameters. This result should be compared to that obtained in the case of type-II SL's in Ref. 15.

The criterion derived above, regarding the existence of acoustic surface plasmons in SL's, is reminiscent of the one found within local nonrelativistic theories for the existence of surface excitations in simpler semi-infinite heterostructures, composed of alternating dielectric slabs with no two-dimensional electron gas in between. ${ }^{28}$ It must be clear, however, that the present discussion [see case (i)] shows that our system also admits surface

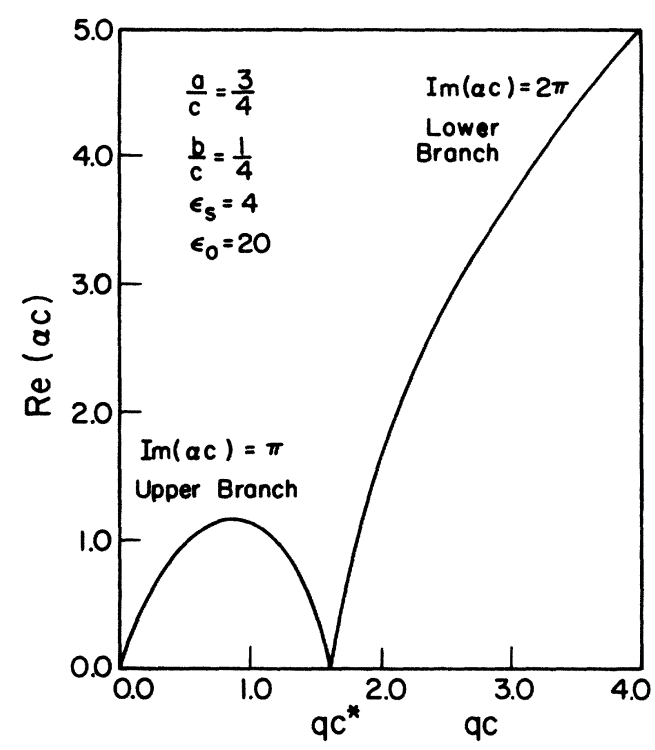

FIG. 7. Plot of $\operatorname{Re}(\alpha c)$, the product of the inverse penetration length and the lattice spacing $c$, versus $q c$ for the two surfaceplasmon branches of Fig. 6. At $q^{*} c$ the penetration length becomes infinite as the surface modes decay into bulk excitations.

plasmons in the case of $a<b$.

Once $\omega_{\mathrm{SP}}(q)$ is known, the complex wave vector $\alpha$ can be calculated via Eq. (9). As it turns out, however, it is simpler to evaluate $\alpha c$ directly from Eq. (3). We set $\eta=\cosh (\alpha c)$, so that

$$
\alpha c=\ln \left[\eta+\operatorname{sgn}(\eta)\left(\eta^{2}-1\right)^{1 / 2}\right],
$$

where, by making use of Eq. (3) in the nonrelativistic limit $i \beta \rightarrow-q$,

$$
\begin{aligned}
\eta= & \left(\gamma^{2} / 4\right)\{\cosh (i \beta c)-\cosh [i \beta(b-a)]\} \\
& -\gamma \sinh (i \beta c)+\cosh (i \beta c) .
\end{aligned}
$$

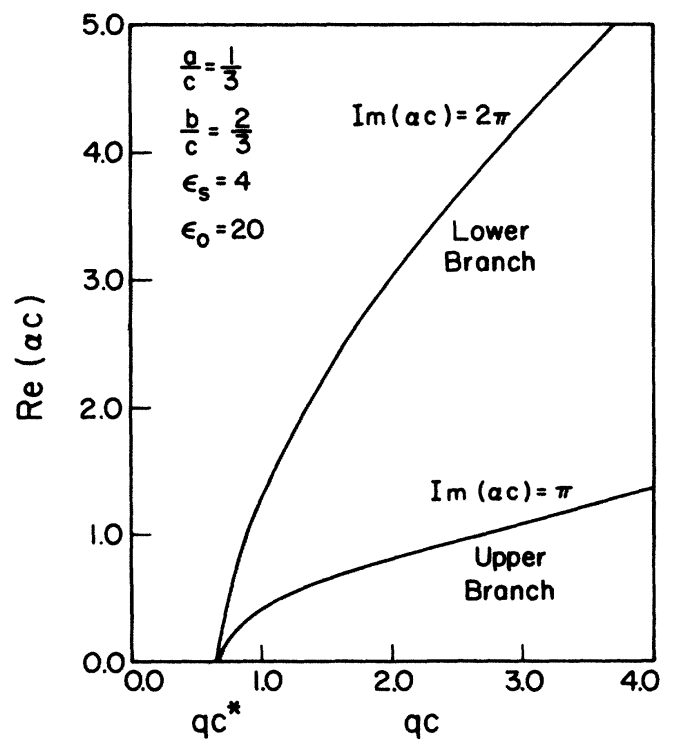

FIG. 8. Same as Fig. 7 for the two surface-plasmon branches of Fig. 4. In this case both modes merge into the continua. 
It is then straightforward, by a direct inspection of $\mathrm{Eq}$. (15), to draw the following conclusions:

(a) When $\omega>\omega_{+}(q, O), \alpha$ has only a positive real part which approaches zero from above as $q \rightarrow q^{*}$, as illustrated in Fig. 7.

(b) For the case of the ESP, i.e., when

$$
\omega_{+}(q, \pi / c)>\omega>\omega_{-}(q, \pi / c),
$$

$\alpha$ acquires an imaginary part equal to $\pi / c$, whereas the real part of $\alpha$ goes to zero both for $q \rightarrow q^{*}$ and for the $a>b$ case, as $q \rightarrow 0$. This is illustrated in Figs. 7 and 8 .

(c) When $\omega<\omega_{-}(q, 2 \pi / c)$, the imaginary part of $\alpha$ is equal to $2 \pi / c$.

(d) When $\omega$ lies inside the bulk continua, $\alpha$ is purely imaginary, so that all the modes are propagating through the structure (see Sec. II).

When the dispersion curve of the surface plasmons merges into the continua, the excitations will decay into bulk modes. At this point, the real part of $\alpha$ goes to zero. This corresponds to an infinite penetration length. The critical value of $q$ where this occurs can be calculated by requiring that $\eta= \pm 1$. After some algebraic manipulations, we obtain

$$
q^{*}=(1 / b) \ln \left|\left(\epsilon_{R}+1\right) /\left(\epsilon_{R}-1\right)\right| .
$$

This result is very similar to that obtained for the case of a type-I SL and reduces to it as $b \rightarrow a .^{10}$

\section{ACKNOWLEDGMENTS}

The authors gratefully acknowledge partial support by National Science Foundation (Materials Research Laboratories Program) Grant No. DMR-84-18453 at Purdue University, and by the U.S. Army Research Office (Durham, NC).
${ }^{1}$ L. Esaki, in Novel Materials and Techniques in Condensed Matter, edited by G. W. Crabtree and P. Vashishta (NorthHolland, New York, 1982), p. 1.

${ }^{2}$ A. L. Fetter, Ann. Phys. (N.Y.) 81, 367 (1973).

${ }^{3}$ W. L. Bloss and E. M. Brody, Solid State Commun. 43, 523 (1982).

${ }^{4}$ S. Das Sarma and J. J. Quinn, Phys. Rev. B 25, 7603 (1982).

${ }^{5}$ A. C. Tselis, G. Gonzales De La Cruz, and J. J. Quinn, Solid State Commun. 46, 779 (1983).

${ }^{6}$ G. Gonzales De La Cruz, A. C. Tselis, and J. J. Quinn, J. Chem. Phys. Solids 44, 807 (1983).

${ }^{7}$ D. Olego, A. Pinczuk, A. C. Gossard, and W. Wiegmann, Phys. Rev. B 25, 7867 (1982).

${ }^{8}$ Z J. Tien, J. M. Worlock, C. H. Perry, A. Pinczuk, R. L. Aggerwal, H. L. Stormer, A. C. Gossard, and W. Weigmann, Surf. Sci. 113, 89 (1982).

${ }^{9}$ G. Fasol, H. P. Hughes, and K. Ploog (unpublished).

${ }^{10}$ G. F. Giuliani and J. J. Quinn, Phys. Rev. Lett. 51, 919 (1983).

${ }^{11}$ R. H. Ritchie, Phys. Rev. 106, 874 (1957).

${ }^{12}$ E. A. Stern and R. A. Ferrell, Phys. Rev. 120, 130 (1960).

${ }^{13}$ D. E. Beck, Phys. Rev. B 4, 1555 (1971).

${ }^{14}$ G. F. Giuliani, Bull. Am. Phys. Soc. 29, 298 (1984).

${ }^{15}$ G. Qin, G. F. Giuliani, and J. J. Quinn, Phys. Rev. B 28, 6144 (1983).

${ }^{16}$ G. F. Giuliani, G. Qin, and J. J. Quinn, Surf. Sci. 142, 433 (1984).

${ }^{17}$ G. F. Giuliani, G. Qin, and J. J. Quinn, in Proceedings of the 17th International Conference on the Physics of Semiconductors, edited by J. D. Chady and W. A. Harrison (Springer-
Verlag, New York, 1985), p. 511.

18J. K. Jain and P. B. Allen, Phys. Rev. Lett. 54, 947 (1985); Phys. Rev. B 32, 5456 (1985).

${ }^{19}$ G. F. Giuliani, G. Qin, and J. J. Quinn (unpublished).

${ }^{20} \mathrm{~A}$ preliminary account of this investigation can be found in $\mathbf{G}$. F. Giuliani and J. J. Quinn, Bull. Am. Phys. Soc. 29, 216 (1984).

${ }^{21}$ For simplicity, we limit our discussion to the intrasubband excitations only. The generalization to the case in which both intrasubband and intersubband transitions are involved is straightforward.

22F. Stern, Phys. Rev. Lett. 18, 546 (1967).

${ }^{23}$ This condition is well verified in all practical cases due to the low electronic density in the layers.

${ }^{24} \mathrm{We}$ have assumed here that we are in the so-called electric quantum limit in which only the lowest electric subband is occupied.

${ }^{25}$ W. L. Bloss, Surf. Sci. 142, 427 (1984).

${ }^{26}$ For simplicity, we will ignore the possibility of a buffer insulating region between the very first layer and the actual interface.

${ }^{27}$ G. F. Giuliani (unpublished); G. F. Giuliani, J. J. Quinn, and R. F. Wallis, J. Phys. (Paris) Colloq. 45, C5-285 (1984).

${ }^{28}$ See R. E. Camley and D. L. Mills, Phys. Rev. B 29, 1695 (1984) for a nonrelativistic approach. A fully relativistic treatment is discussed in R. Szenics, R. F. Wallis, G. F. Giuliani, and J. J. Quinn, Surf. Sci. 166, 45 (1986). In the general case, surface-plasmon polaritons can exist also when the condition $a>b$ is violated. 